\title{
Trabeculectomy training in England: are we safe at training? Two year surgical outcomes
}

\author{
A. Walkden ${ }^{1} \cdot$ J. Huxtable ${ }^{1} \cdot$ M. Senior ${ }^{1} \cdot$ H. Lee ${ }^{1} \cdot$ S. Naylor ${ }^{1} \cdot$ S. Turner ${ }^{2} \cdot K_{\text {K Ivanova }}^{2} \cdot$ J. Koppens $^{3} \cdot$ B. Todd ${ }^{3}$.

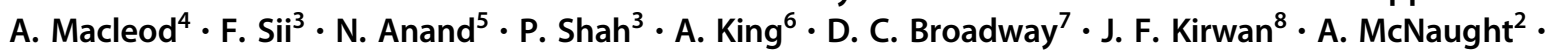 \\ A. Bhan-Bhargava ${ }^{9}$
}

Received: 8 May 2017 / Revised: 25 August 2017 / Accepted: 3 February 2018 / Published online: 12 March 2018

(c) The Royal College of Ophthalmologists 2018

\begin{abstract}
Objectives To define the safety profile of trainee trabeculectomy surgery in the United Kingdom. Surgical exposure for trainees in England is limited due to service requirements, the European working time directive constraints and increasing sub-specialisation of glaucoma surgery. Limited knowledge exists on the outcomes of supervised glaucoma surgery. The aim is to determine the safety of supervised trabeculectomy surgery performed by trainee ophthalmologists.

Methods Retrospective case note review of all patients that had trabeculectomy surgery with MMC by consultant and trainee surgeons across multiple UK centres. All eyes have 2-year follow up. Success was determined using WGA guidelines. Two-tailed $p$ values were obtained using Fisher's exact test to ascertain statistical significance between groups. Main outcome measures: intraocular pressure, visual acuity, success and failure rates.

Results 324 eyes were reviewed. 211 (66.4\%) cases were performed by glaucoma consultants, 107(33.6\%) by trainee ophthalmologists. The majority of eyes in each group were undergoing surgery for POAG. Post-operative IOP control showed no significant difference between consultant and trainee groups at year 1 and year 2. Success rates showed no significant difference between consultant and trainee cases. Failure rates at year 1 showed a significant difference between the two groups. No significant difference was seen at year 2 . The trainee group had significantly more complications, when compared with the consultant group. Snellen visual acuity loss was not statistically significant between the two groups at the 2 year time point. Conclusions The outcomes of supervised trainee trabeculectomy compare favourably with consultant cases after 2 year follow up. Trainee cases had higher complication rates than consultant cases. Bleb leaks are a common complication of trainee cases, where closer supervision may be required. There is potential for surgical simulation to help increase the success of such cases. These findings may encourage trainee participation in glaucoma surgery.
\end{abstract}

\section{Introduction}

Trabeculectomy rates have fallen steadily over the last few decades. The advent of more effective topical medications has resulted in less patients requiring trabeculectomy [1]. The fall in glaucoma filtration surgical rates, coupled with the trend towards sub-specialisation and the constraints of the European Working Time Directive (EWTD) [2], has
A. Walkden

walkdenandrew@gmail.com

1 Department of Ophthalmology, Lancashire Teaching Hospitals, Preston, UK

2 Gloucestershire Hospitals NHS Foundation Trust, Cheltenham, UK and Cranfield University, Bedford, UK

3 University Hospital Birmingham NHS Foundation Trust, Birmingham, UK

4 Southampton General Hospital, Southampton, UK
5 Calderdale \& Huddersfield NHS Foundation Trust, Huddersfield, UK

6 Nottingham University Hospital, Queens Medical Centre Campus, Nottingham, UK

7 Norfolk and Norwich University Hospital \& University of East Anglia, Norwich, UK

8 Queen Alexandra Hospital, Portsmouth, UK

9 Aintree University Hospital, Liverpool, UK 
resulted in increasingly limited trainee exposure to trabeculectomy [3]. With greater patient expectations and an increasingly litigious climate, trabeculectomy training opportunities for trainees can be limited. Concerns have been raised by previous authors regarding the bias towards service delivery rather than surgical training [2]. The problems related to glaucoma filtration surgery training are likely to be exacerbated further if plans to shorten the length of specialist training in the UK are implemented [4]. In addition, informing patients accurately during the consenting process can be difficult as limited knowledge exists on the outcomes and safety of trabeculectomy surgery performed by trainees.

Trabeculectomy has long been considered a vital component of ophthalmic surgical training. Prior to modern phacoemulsifaction cataract surgery it was suggested as a method of introduction to intraocular surgery [5] because of the variety of techniques that can be acquired from the different steps of the operation, such as tissue handling and suturing. However, the current Royal College of Ophthalmologists (RCOphth) curriculum suggest trainees should be able to 'undertake surgical measures to lower intraocular pressure', with this learning outcome encompassing many different surgical interventions. However, performing a trabeculectomy case is not a prerequisite for the completion of specialist training, unlike phacoemulsification surgery, for example.

With these factors in mind, the authors of this paper set out to evaluate the outcomes and safety of trabeculectomies performed by trainees under consultant supervision in England, compared with the outcomes of fellowship trained glaucoma consultants. We report a multicentre case series of 324 eyes to determine the outcomes and safety of trainee trabeculectomy outcomes.

\section{Methods}

A review of 324 consecutive primary trabeculectomies augmented with mitomycin-c (MMC) was undertaken with data provided from seven English centres (Lancashire Teaching Hospital, Gloucestershire Hospital, Calderdale and Huddersfield Hospital, Queen Alexandra Hospital Portsmouth, Birmingham University Hospitals, Birmingham and Midlands Eye Centre, Nottingham University Hospitals \& Norfolk and Norwich University Hospital), between 2002 and 2013. All included patients had at least 2 years of follow up. Only eyes with the following previous ophthalmic interventions were included: (1) uncomplicated phacoemulsification surgery, (2) laser peripheral iridotomy or (3) laser trabeculoplasty. All centres provided surgical training to specialist registrars or equivalent ophthalmic trainees, supervised by a fellowship trained glaucoma consultant. All data was then collated centrally, with any data queries being confirmed by the providing centre.

All trabeculectomies were performed either by a fellowship trained glaucoma consultant or a trainee under the direct supervision of a scrubbed glaucoma consultant. In all trainee cases, the trainee was the primary surgeon, carrying out the operation with direct assistance from the consultant if required.

Although a range of surgeons were involved in the data series, all trabeculectomies were performed using a fornix based conjunctival flap, rectangular scleral flap, punch sclerostomy, peripheral iridotomy and releasable sutures to the scleral flap. All cases used mitomycin-C. (MMC). All patients were reviewed day 1 and week 1 post operatively and thereafter based on clinical need.

Participating glaucoma specialists populated an Excel spreadsheet (Microsoft Corp, Redmond, WA) collecting predefined data. For confidentiality purposes, each patient and consultant were coded. All data was stored in accordance with the Data Protection Act 1998. At all visits, Snellen visual acuity was recorded along with intraocular pressure (IOP) using Goldmann applanation tonometry. Data was collected as per the World Glaucoma Association (WGA) Guidance on to reporting surgical trials [6], including pre-operative IOP, IOP at 1 year, IOP at 2 years and post-operative requirement for topical medication. All intra-operative and post-operative complications were recorded.

Surgical outcomes were then classified as per the WGA guidance into Complete Success, Qualified success and Failure. Complete success was defined as an IOP of less than $21 \mathrm{~mm} \mathrm{Hg}$ on no topical or oral IOP lowering medication. Qualified success was defined as an IOP of less than $21 \mathrm{~mm} \mathrm{Hg}$ with adjunctive use of one or more topical IOP lowering agents.

Trabeculectomy failure was defined as one or more of the following;

(1) Loss of $\geq 2$ lines of Snellen acuity at 1 or 2 years.

(2) Surgical Complications or a further operation (but not including needling, antimetabolite injections or removal of releasable sutures).

(3) IOP $>21 \mathrm{~mm} \mathrm{Hg}$ (even with topical medication) at 1 or 2 years.

Two-tailed Fisher's exact tests were used to assess differences in categorical data. Paired Student's t-tests were used to assess differences in continuous data. $P$ values of $<0.05$ were considered statistically significant. All data was managed with Excel (Microsoft Corp) and analysed using both Excel and GraphPad Prism software (GraphPad Software, Inc., La Jolla, CA). 


\section{Results}

324 eyes were reviewed after data was received from all centres. 6 cases $(1.85 \%)$ were excluded due to incomplete follow up data, leaving a total of 318 eyes for analysis. Mean patient age was 72.7 years (range 35-98 years). 211 (66.4\%) cases were performed by fellowship trained glaucoma consultants, 107 (33.6\%) performed by trainee ophthalmologists with 3-7 years of surgical experience. This includes 'fellows' who are undergoing higher subspecialty training after completing general ophthalmic training. All trainees had completed a minimum of 3 years specialist training and had previous surgical experience which was predominantly cataract surgery, minor lid operations and assisting with more complex specialist cases but limited experience of trabeculectomy.

Patient demographics and the indications for undergoing trabeculectomy surgery in both groups are shown in Table 1. The majority of eyes in each group were undergoing surgery for POAG, with 154 eyes $(73.0 \%)$ in the consultant group and 80 eyes (74.8\%) in the trainee group. Pre-operative IOP was similar amongst the two groups.

Post-operative IOP control showed no significant difference between consultant and trainee groups at year 1 and year $2(\mathrm{p}=0.88$ and 0.61 respectively). Table 2 shows IOP outcomes for both groups at year 1 and year 2 . Success rates at year 1 and year 2 showed no significant difference between consultant and trainee cases $(p=0.22$, 0.53 respectively). Failure rates differed significantly in year 1 , but did not by the end of year $2(p=0.04)$. No significant difference was seen at year $2(p=0.50)$. Table 3 shows the success, qualified success and failure rates of both groups.

Table 1 Patient demographics

\begin{tabular}{|c|c|c|}
\hline & Consultant & Trainee \\
\hline \multirow[t]{2}{*}{ Cases per group $(n=)$} & 211 & 107 \\
\hline & $\begin{array}{l}211 \text { (glaucoma } \\
\text { specialist) }\end{array}$ & $\begin{array}{c}18 \text { (fellow) } 89 \\
\text { (trainee) }\end{array}$ \\
\hline $\operatorname{Sex}(n=)$ & $124(58.8 \%)$ & 65 female $(60.7 \%)$ \\
\hline Mean age (years) & 72.0 & 73.4 \\
\hline Age range (years) & $35-98$ & $43-93$ \\
\hline $\begin{array}{l}\text { Mean pre op IOP (mm } \\
\mathrm{Hg}) \text { (range) }\end{array}$ & $24.0(11-56)$ & $23.1(8-60)$ \\
\hline \multicolumn{3}{|l|}{ Diagnosis } \\
\hline POAG/OHT $(n=)$ & $154(73.0 \%)$ & $80(74.8 \%)$ \\
\hline NTG & $8(3.79 \%)$ & $7(6.54 \%)$ \\
\hline CACG & $18(8.53 \%)$ & $11(10.3 \%)$ \\
\hline PXF/PDS & $11(5.21 \%)$ & $4(3.74 \%)$ \\
\hline Uveitic & $7(3.32 \%)$ & $1(0.94 \%)$ \\
\hline Other & $13(6.16 \%)$ & $4(3.74 \%)$ \\
\hline
\end{tabular}

Complications occurring within the 2 year follow up period are shown in Table 4. 94.9\% $(n=37)$ of the complications were post operative. $5.1 \%(n=2)$ occurred intraoperatively (iris prolapse and aqueous misdirection). Bleb leak and hyptony were the most common complications for both groups ( $n=15$ for each). The trainee group had more complications, with statistical significance, when compared with the consultant group $(p=0.02)$. The only significant difference was for bleb leaks but if other possible complications were excluded, no difference in complication rates was found between the two groups $(\mathrm{p}=0.38)$.

Snellen visual acuity loss of $\geq 2$ lines was not statistically significant between the two groups at the 2 year time point $(p=0.41)$, but was significant at year 1 , being higher in trainee cases $(p=0.01)$. No statistical significance was seen between the two groups in terms of patients requiring topical medication at both year 1 and year 2 .

\section{Discussion}

Limited data exists on the outcomes of surgery performed by ophthalmic trainees in England. The aim of the present study was to determine the safety and efficacy of trabeculectomy performed by trainees in England using a large, multicentre cohort of patients. We have shown that there is no statistically significant difference in IOP between consultant and trainee operations at both 1 and 2 year follow up. Complete success and qualified success rates were similar amongst both groups and no statistically significant difference was demonstrated throughout the 2 year follow up period.

When considering year 1 and year 2 data separately, the trainee group had a statistically significantly higher failure rate when compared with the consultant group at year 1 follow up. At year 2, the trainee failure rate was less than at year 1 and was similar between the consultant and trainee groups with no statistically significant difference. The findings might have been due to patients being started on topical treatment after 1 year or undergoing bleb needling.

Over the 2 year period the trainee group had a statistically significant higher complication rate, with bleb leaks and hypotony being the most frequent complications. This chimes with the findings of Welch et al. who have shown previously that trainee trabeculectomies have higher return to theatre rates associated with bleb leaks [7]. Interestingly in their subgroup analysis of the MMC trabeculectomies they also found trainee cases had significantly higher return to theatre rates. In an Australian surgical cohort, Sun and Lee [8] found trainee trabeculectomies to have a similar rate of bleb leak when compared with consultant outcomes. The findings of Welch et al. and Sun and Lee have been 
Table 2 Outcomes year 1 and year 2

\begin{tabular}{lllllll}
\hline Surgeon Grade & $\begin{array}{l}\text { Mean post op IOP } \\
(\mathrm{mm} \mathrm{Hg} ; n=)\end{array}$ & $\begin{array}{l}\mathrm{IOP}<21 \\
(n=)\end{array}$ & $\begin{array}{l}\mathrm{IOP}<16 \\
(n=)\end{array}$ & $\begin{array}{l}\text { IOP }>6 \\
(n=)\end{array}$ & $\begin{array}{l}\text { Patients requiring topical } \\
\text { medication }(n=)\end{array}$ & $\begin{array}{l}>2 \text { line snellen } \\
\text { acuity drop }(n=)\end{array}$ \\
\hline $\begin{array}{l}\text { Trainee group: year 1 } \\
\text { 13.0 (SD =4.90) }\end{array}$ & $100(93.5 \%)$ & $89(83.2 \%)$ & $105(98.1 \%)$ & $7(6.54 \%)$ & $11(10.3 \%)$ \\
$\begin{array}{l}\text { Consultant group: } \\
\text { year 1 }\end{array}$ & $12.5(\mathrm{SD}=4.12)$ & $202(95.7 \%)$ & $37(82.5 \%)$ & $210(99.5 \%)$ & $18(8.5 \%)$ & $6(2.84 \%)$ \\
$\begin{array}{l}\text { Trainee group: year 2 } \\
\text { Consultant group: }\end{array}$ & $13.0(\mathrm{SD}=4.1)$ & $105(98.1 \%)$ & $87(81.3 \%)$ & $106(99.1 \%)$ & $14(13.1 \%)$ & $13(12.2 \%)$ \\
year 2 & $12.5(\mathrm{SD}=4.31)$ & $206(97.6 \%)$ & $167(79.1 \%)$ & $206(97.6 \%)$ & $28(13.3 \%)$ & $34(16.1 \%)$ \\
\hline
\end{tabular}

Table 3 Outcomes at year endpoints: consultant and trainee cases

\begin{tabular}{|c|c|c|c|c|c|c|c|}
\hline Surgeon gade & Complete success $(n=)$ & $P=$ & Qualified Success $(n=)$ & $P=$ & Failure $(n=)$ & $P=$ & Total $(n=)$ \\
\hline Trainee group: year 1 & $76(71.00 \%)$ & & $2(1.87 \%)$ & & $29(27.10 \%)$ & & 107 \\
\hline Consultant group: year 1 & $164(77.7 \%)$ & 0.22 & $11(5.21 \%)$ & 0.23 & $36(17.1 \%)$ & 0.04 & 211 \\
\hline Trainee group: year 2 & $73(68.2 \%)$ & & $9(8.41 \%)$ & & $25(23.4 \%)$ & & 107 \\
\hline Consultant group: year 2 & $135(64.0 \%)$ & 0.53 & $18(8.53 \%)$ & 1.00 & $58(27.5 \%)$ & 0.50 & 211 \\
\hline
\end{tabular}

$P$ values (3 sig fig) looking for statistical significance between consultant and trainee groups

Table 4 Complications occurring within 2 years

\begin{tabular}{|c|c|c|c|c|}
\hline Complication & $\begin{array}{l}\text { Consultant } \\
\text { Group }(n=)\end{array}$ & $\begin{array}{c}\text { Trainee } \\
\text { Group }(n=)\end{array}$ & Total $(n=)$ & $P=$ \\
\hline Hyphaema & 2 & 3 & 5 & - \\
\hline Hypotony & 9 & 6 & 15 & - \\
\hline Bleb leak & 5 & 10 & 15 & - \\
\hline $\begin{array}{l}\text { Intra-operative } \\
\text { aqueous } \\
\text { misdirection }\end{array}$ & 1 & 0 & 1 & - \\
\hline Iris Prolapse & 0 & 1 & 1 & - \\
\hline Blebitis & 1 & 0 & 1 & - \\
\hline Endophthalmitis & 1 & 0 & 1 & 一 \\
\hline Total & $19(9.0 \%)$ & $20(18.7 \%)$ & $39(12.5 \%)$ & 0.02 \\
\hline $\begin{array}{l}\text { Total with bleb } \\
\text { leaks excluded }\end{array}$ & $14(6.64 \%)$ & $10(9.34 \%)$ & $24(7.5 \%)$ & 0.38 \\
\hline
\end{tabular}

supported by those of Broadway and Clark [9], although their study reported increased rates of hypotonous maculopathy amongst the trainee bleb leak cases. The findings from the present study showed that both consultant and trainee groups had lower bleb leak rates $(2.4 \%$ and $9.35 \%$ respectively) when compared with another large UK national survey of trabeculectomy performed by consultants with 1 year follow up [10]. The largest, most recent survey of UK trabeculectomy outcomes published in 2013, Trabeculectomy in the $21^{\text {st }}$ Century authored by Kirwan et al. [11] reported similar outcomes in terms of success and complications to those shown in the present study. However, the Kirwan et al series included 5-FU cases and a small number of non-antimetabolite augmented trabeculectomies. Similar, but not identical measures of success and failure were used. Both supervised trainee and consultant outcomes compared favourably, with the aforementioned study which reported a bleb leak complication rate of $14 \%$. In addition, our endophthalmitis rate $(0.47 \%$ in the consultant group) was similar to that reported by Kirwan et al $(0.5 \%)$. Our data showed lower rates of endophthalmitis and blebitis in both groups when compared with the Tube vs Trabeculectomy 3 year outcomes paper [12]. Broadway and Clark [9] looked at trabeculectomy outcomes with a mean follow up duration of 5 years and sub analysed their trainee cases. Trainee cases were found to be a risk factor for increased trabeculectomy complications, post-operative bleb manipulation and less successful outcomes. The findings of the present study were in agreement in terms of failure and complication rates, although we found no statistically significant difference in overall success after 2 years of follow up, when compared with consultant outcomes. It must be stressed however that it is difficult to compare our findings directly with those of Broadway and Clark's [9], since their cases had a longer follow up period which might have led to a higher failure rate and cases that had a bleb needling carried out after 3 months were considered as failures. Furthermore, the present study looked specifically at MMC augmented trabeculectomies, which have been shown to increase post-operative success [9, 11] compared to 5-FU augmented and unaugmented trabeculectomy. Finally, the 'trainee group' in the Broadway and Clark study had a larger proportion of 'fellows' (39\% specifically training in glaucoma) performing cases than for 
the present study 'trainee' cohort, which may have had an affect on overall success rates.

Importantly, our results and those of other authors have shown that post-operative IOP control tends to be similar amongst the two groups at the 2 year follow up time point $[7,8]$, suggesting that supervised trainee trabeculectomy is not associated with reduced efficacy when compared with the outcomes of fellowship trained glaucoma consultants.

In the present study decreases in visual acuity at year 1 were higher in the trainee group, showing a statistically significant difference when compared with the consultant cases. Although the causes for decreases in visual acuity were not collected as part of the study, the authors considered it most likely that this was due to either postoperative refractive change or cataract formation and not necessarily progression of glaucomatous optic neuropathy. Our study found lower rates of visual loss than in The National Survey of Trabeculectomy study, which found visual loss of more than one Snellen line to occur in $18.8 \%$ of cases after one year of follow up in a large cohort of patients [10]. In addition, a recent retrospective study from the US found post trabeculectomy visual acuities to be significantly reduced amongst eyes operated on by resident surgeons as opposed to attending surgeons with a 2 year follow up, albeit only using a cohort of only 17 and 21 eyes, respectively [13].

From a training perspective the present study has supported other literature in demonstrating higher rates of postoperative bleb leak in trabeculectomies performed by trainees. Conjunctival flap suturing must therefore be targeted as an area for improvement, whether it be with intensive simulated suturing skills prior to operating on live patients or the consultant 'taking over' or 'double checking' this part of the operation. Although most bleb leaks were managed conservatively with no significant intervention required, if suturing skills were to be improved amongst the trainee group, surgical outcomes would be likely to improve to a level comparable with consultants. With bleb leaks removed from the complications analysis, no significant difference existed between the two groups. Unlike previous generations [5], today's trainees are introduced to modern phacoemulsification surgery that routinely requires no suturing. Limited experience in microscopic suturing skills is likely to be a contributing factor as to why conjunctival closure is found to be difficult by trainees reflected in their higher rate of bleb leak. With the ever increasing numbers of surgical simulation tools available, the authors feel strongly that these can be used to improve basic surgical skills.

Due to a shift within the NHS towards consultant delivered care it is vital that future generations of ophthalmic surgeons are able to access surgical training opportunities. It is of paramount importance that these opportunities are delivered in a way that ensures safety and quality outcomes to our patients. Excellent surgical simulation techniques have been developed which help trainees to develop basic surgical techniques, and plans are in place to include these as part of a modular learning component to the current RCOphth curriculum [14]. Simulated techniques have been shown to increase competency in cataract surgery [15]. Specific to glaucoma surgery, other authors have developed wet lab techniques using pig eyes [16] and advanced model surgical simulation eyes [17]. Simulated models provide trainees with a stress free educational environment and affords the opportunity for technique replication that 'real life', busy theatre environments do not permit. Although excellent for building surgical foundations, simulation cannot replace the experience gained with real life tissue handling. Other authors have surveyed Scottish consultant ophthalmologists and they are of the opinion that higher specialist trainees should continue to gain exposure to trabeculectomy surgery [7]. The findings of our study support the safety of supervised trabeculectomy performed by trainee ophthalmologists as part of their training.

The present study has provided much needed, multicentre results of trainee trabeculectomies augmented with MMC, using the largest dataset of cases performed by trainees within the current literature. Trainees compared favourably with other large national studies $[10,11]$ as well as other reported trainee outcomes [7-9].

Limitations exist with any retrospective study, although our data capture was good at $98.2 \%$. Our study used 2 year outcomes which may not truly reflect the longer term function of the trabeculectomies, although longer follow up data on trainee cases within the literature is scarce $[9,18]$. As previously discussed, the aim of our study was to determine the success of trainee trabeculectomy outcomes with a 2 year follow up. By doing this, we have provided collateral data on consultant outcomes as a means of comparison. As would be the case with any 'real world dataset' it would not be unreasonable to expect that consultants performed the surgery for more difficult cases, thereby affecting the surgical outcomes and adding limitations to the direct comparison of the outcomes of each group.

It is worth considering the well documented fact that posterior capsular rupture rates are higher amongst junior cataract surgeons when compared with experienced surgeons $[19,20]$. Given that cataract surgery remains the mainstay of modern ophthalmic surgical training, this would suggest that there is some tolerance of a 'training cost' in terms of outcome quality. However, this discussion is well beyond the scope of this article, even with all of the above factors taken into account. In the present study it was reassuring that IOP control and visual acuity outcomes were acceptable and similar or better than the findings of previous authors $[3,7,8,10,11]$. Our observation of a higher failure rate at year 1 follow up in the trainee group and the 
higher rates of bleb leak was significant; one might consider this analogous to contemporary cataract surgery where trainee surgeons are associated with higher rates of posterior capsular compared with experienced surgeons [19, 20].

It is important to present the alternative side to the above argument. With the micro-invasive era of glaucoma surgery (MIGS) now well underway, it may well be that in the near future these procedures are performed in conjunction with routine cataract surgery by non-glaucoma specialists. Depending on their efficacy, this approach may therefore limit trabeculectomy to be performed solely by glaucoma specialists. It can therefore be argued that trabeculectomy should only be taught to trainee surgeons committed to becoming a glaucoma subspecialist.

Finally, with patients becoming better informed and more knowledgeable about their conditions it means we can now use the results of this study to help to more accurately inform our patients during the consenting process if their surgery is to be performed by trainees. We have also highlighted bleb formation and conjunctival suturing as an area that can be targeted to improve trainee trabeculectomy outcomes and safety in the future; if these areas were to be addressed they could potentially improve the already favourable outcomes of trainee undertaken trabeculectomy.

\section{Summary}

\section{What was known before}

- Trabeculectomy surgery is the main surgical intervention to halt progression of glaucoma. Increasing subspecialisation can lead to reduced surgical exposure to trainee surgeons. The safety profile of modern trabeculectomy performed by trainees has not been defined.

\section{What this study adds}

- Supervised trainee trabeculectomy outcomes have a good safety profile. Improving trainee suturing techniques may lead to better surgical outcomes. Defining the safety profile of trainee surgery can help aid the consent process for patients undergoing surgery.

\section{Compliance with ethical standards}

Conflict of interest The authors declare that they have no conflict of interest.

\section{References}

1. Fraser SG, Wormald RPL. Hospital Episode Statistics and changing trends in glaucoma surgery. Eye. 2007; 22:3-7.

2. Gibson A, Boulton M, Watson M, Fielder A. Surgical training in ophthalmology. Lancet. 2002;360(Issue 9346): P 1702.

3. Troutbeck R, Lee GA, Sanfilippo P, Fleming B. Trabeculectomy: a training dilemma. Eye. 2007;21:183-86.

4. Shape of training report. Final Report by Prof Greenaway http://www.shapeoftraining.co.uk/static/documents/content/Sha pe_of_training_FINAL_Report.pdf_53977887.pdf.

5. Morrell AJ, Searle AE, O'Neill EC. Trabeculectomy as an introduction to intraocular surgery in an ophthalmic training program. Ophthalmic Surg. 1989;20:557-60.

6. Shaarawy TM, Grehn F, Sherwood MB, eds. WGA guidelines on design and reporting of glaucoma surgical trials. Amsterdam, The Netherlands: Kugler 2008; p. 15-24. 26

7. Welch $J$, et al. Glaucoma surgery: trainee outcomes and implications for future training: Southeast Scotland. Eye. 2010;24:1700-7.

8. Sun L, Lee GA. Trabeculectomy: the limitations for registrar training. J Clin Exp Ophthalmol. 2013;41:135-9.

9. Broadway DC, Clark A. The Norwich trabeculectomy study: long-term outcomes of modern trabeculectomy with respect to risk factors for filtration failure. J Clin Exp Ophthalmol. 2014:5:371.

10. Edmunds, et al. The national survey of trabeculectomy III: early and late complications. Eye. 2002;16:297-303.

11. Kirwan, et al. Trabeculectomy in the $21^{\text {st }}$ century: multicentre analysis. Ophthalmology. 2013;120:2532-9.

12. Gedde J, et al. Three-year follow-up of the tube versus trabeculectomy study. Am J Ophthalmol. 2009;148: 670-84.

13. Kwong, et al. Long-term outcomes of resident- versus attendingperformed primary trabeculectomy with mitomycin $\mathrm{C}$ in a United States residency program. Am J Ophthalmol. 2014;157:1190-201.

14. RCOphth Training Curriculum - eportfolio website. www. rcophth.co.uk.

15. Bergqvist J, Person A, Vestergaard A, Grauslund J. Establishment of a validated training programme on the Eyesi cataract simulator. A prospective randomized study. Acta Ophthalmol (Copenh). 2014;92:629-34.

16. Lee GA, Chiang MY, Shah P. Pig eye trabeculectomy: a wet-lab teaching model. Eye. 2006;20:32-7.

17. -Trabeculectomy Advanced Eyes Website - http://simulatedocula rsurgery.com/product/trabeculectomy-eye-advanced.

18. Landers, et al. 20 year follow up of trabeculectomy: risk factors and outcomes. Ophthalmology. 2012;119:694-702.

19. Sparrow JM, Taylor H, Qureshi K, Smith R, Birnie K, Johnston RL, the UK EPR user group. The Cataract National Dataset electronic multi-centre audit of 55567 operations: risk indicators for monocular visual acuity outcomes. Eye. 2012;26:821-6.

20. Buchan JC, Cassels-Brown A. Determinants of cataract surgical opportunities and complication rates in UK higher specialist training. Eye. 2008;22:1425-9. 